\title{
Limit cycle bifurcations and analytic center conditions for a class of generalized nilpotent systems
}

\author{
Yusen $\mathrm{Wu}^{\mathrm{a}, *}$, Cui Zhang ${ }^{\mathrm{b}}$, Sumin Yang ${ }^{\mathrm{c}}$ \\ a School of Mathematics and Statistics, Henan University of Science and Technology, Luoyang, 471023 Henan, P. R. China. \\ ${ }^{b}$ School of Mathematical Science, Luoyang Normal University, Luoyang, 410022 Henan, P. R. China. \\ ${ }^{c}$ School of Humanities and Science, Guangxi Technological College of Machinery and Electricity, Nanning, 530007 Guangxi, P. R. \\ China.
}

\begin{abstract}
Bifurcation of limit cycles and analytic center conditions for a class of systems in which the origin is a generalized nilpotent singular point are discussed. An interesting phenomenon is that the exponent parameter $n$ controls the singular point type of the studied system (1.1). (C)2017 All rights reserved.
\end{abstract}

Keywords: Limit cycle bifurcation, analytic center conditions, generalized nilpotent systems. 2010 MSC: 34C05, 37G15.

\section{Introduction}

In the present paper, we consider limit cycle bifurcations and analytic center conditions of the origin for a class of generalized nilpotent systems with the form

$$
\begin{aligned}
& \frac{d x}{d t}=y^{2 n-1}+y^{n-1}\left(a_{41} x^{4} y^{n}+a_{32} x^{3} y^{2 n}+a_{23} x^{2} y^{3 n}+a_{14} x y^{4 n}+a_{05} y^{5 n}\right) \\
& \frac{d y}{d t}=-2 x^{3}+b_{50} x^{5}+b_{41} x^{4} y^{n}+b_{32} x^{3} y^{2 n}+b_{23} x^{2} y^{3 n}+b_{14} x y^{4 n}+b_{05} y^{5 n}
\end{aligned}
$$

When $n>1$, the origin is a total degenerate singular point. When $n=1$, system (1.1) is rewritten as the following quintic nilpotent systems

$$
\begin{aligned}
& \frac{d x}{d t}=y+a_{41} x^{4} y+a_{32} x^{3} y^{2}+a_{23} x^{2} y^{3}+a_{14} x y^{4}+a_{05} y^{5} \\
& \frac{d y}{d t}=-2 x^{3}+b_{50} x^{5}+b_{41} x^{4} y+b_{32} x^{3} y^{2}+b_{23} x^{2} y^{3}+b_{14} x y^{4}+b_{05} y^{5}
\end{aligned}
$$

whose origin is a third-order nilpotent singular point.

In suitable coordinate system, an analytic ordinary differential equation with an isolated nilpotent singularity at the origin can be written as

$$
\begin{aligned}
& \frac{d x}{d t}=-y+X(x, y), \\
& \frac{d y}{d t}=Y(x, y),
\end{aligned}
$$

\footnotetext{
*Corresponding author

Email address: wuyusen621@126.com (Yusen $\mathrm{Wu})$
}

doi:10.22436/jmcs.017.02.09 
where $X(x, y)$ and $Y(x, y)$ are analytic functions without constant nor linear terms defined in a certain neighborhood of the origin.

The nilpotent center problem was theoretically solved by Moussu [15] and Sadovskii [16]. In order to obtain the local phase portraits, Andreev [2] studied analytic systems with a nilpotent singular point at the origin. However, Andreev's results did not distinguish between a focus and a center. Takens [17] provided a normal form for nilpotent center of foci. Moussu [15] found the $e^{\infty}$ normal form for analytic nilpotent centers. Berthier and Moussu in [3] studied the reversibility of the nilpotent centers. Teixeira and Yang [18] analyzed the relationship between reversibility and the center-focus problem for systems

$$
\begin{aligned}
& \dot{x}=-y+X(x, y), \\
& \dot{y}=x+Y(x, y),
\end{aligned}
$$

and

$$
\begin{aligned}
& \dot{x}=y+X(x, y), \\
& \dot{y}=Y(x, y) .
\end{aligned}
$$

Giné [8] developed a method which provides necessary conditions for obtaining a local analytic integral in a neighborhood of a generalized nilpotent singular point. García and Giné [5] gave a necessary condition to have local analytic integrability in an analytic nilpotent center. Liu and Li [13] gave a recursive method to calculate quasi-Lyapunov constants at the nilpotent critical point. Han and Romanovski [9] studied analytic properties of the Poincaré return map and generalized focal values of analytic planar systems with a nilpotent focus or center. For third-order nilpotent singular points of a planar dynamical system, Liu et al. [14] completely solved the analytic center problem using the integrating factor method. A method to compute focal values for degenerate critical point of switching systems was proposed in [10]. $\mathrm{Li}$ et al. discussed the analytic integrability of two classes of lopsided systems in [11]. In [12], an existing method was modified for computing the focal values and period constants of switching systems associated with elementary singular points. In $[6,7]$ it is proved that any analytic nilpotent center is limit of an analytic linear type center.

Nevertheless, in practice, in spite of the efforts in the last years, given an analytic system with a monodromic point, it is very difficult to know if it is a focus or a center, even in the case of polynomial systems of a given degree. To understand the profound nature of this problem, see [4].

We now describe more precisely different sections of this paper. In Section 2, we give some preliminary knowledge concerning the nilpotent critical point. In Section 3, we transfer the origin into a third-order nilpotent singular point by a homeomorphism. Then we compute the first seven quasi-Lyapunov constants and derive the sufficient and necessary conditions for the origin to be an analytic center. In the last section, we prove that there exist five small amplitude limit cycles created from a nilpotent singular point.

\section{Calculation of quasi-Lyapunov constant and criterion of analytic center}

In this section we first introduce some definitions, notations and symbols in order to make it compact and clear, followed by an algorithm to obtain the necessary conditions for the third-order nilpotent singular point to be an analytic center, then we present several methods to prove the sufficiency due to $[13,14]$.

In canonical coordinates the Lyapunov system with the origin as a nilpotent critical point can be written in the form:

$$
\begin{aligned}
& \frac{d x}{d t}=y+\sum_{i+j=2}^{\infty} a_{i j} x^{i} y^{j}=X(x, y), \\
& \frac{d y}{d t}=\sum_{i+j=2}^{\infty} b_{i j} x^{i} y^{j}=Y(x, y) .
\end{aligned}
$$

Suppose that the function $y=y(x)$ satisfies $X(x, y)=0, y(0)=0$. Lyapunov proved (see for instance [1]) 
that the origin of system (2.1) is a monodromic critical point (i.e., a center or a focus) if and only if

$$
\begin{aligned}
& Y(x, y(x))=\alpha x^{2 n+1}+o\left(x^{2 n+1}\right), \alpha<0, \\
& {\left[\frac{\partial X}{\partial x}+\frac{\partial Y}{\partial x}\right]_{y=y(x)}=\beta x^{n}+o\left(x^{n}\right),} \\
& \beta^{2}+4(n+1) \alpha<0
\end{aligned}
$$

where $n$ is a positive integer.

Definition 2.1. Let $y=f(x)=-a_{20} x^{2}+o\left(x^{2}\right)$ be the unique solution of the function equation $X(x, f(x))=$ $0, f(0)=0$ at a neighborhood of the origin. If there are an integer $m$ and a nonzero real number $\alpha$, such that

$$
Y(x, f(x))=\alpha x^{m}+o\left(x^{m}\right),
$$

we say that the origin is a high-order singular point of system (2.1) with the multiplicity $m$.

By using the results in [1], we attain the following conclusion.

Lemma 2.2. The origin of system (2.1) is a third-order singular point which is a saddle point or a center, if and only if $\mathrm{b}_{20}=0,\left(2 \mathrm{a}_{20}-\mathrm{b}_{11}\right)^{2}+8 \mathrm{~b}_{30}<0$.

When the condition in Lemma 2.2 holds, we can assume that

$$
a_{20}=\mu, \quad b_{20}=0, \quad b_{11}=2 \mu, \quad b_{30}=-2 .
$$

Otherwise, by letting $\left(2 a_{20}-b_{11}\right)^{2}+8 b_{30}=-16 \lambda^{2}, 2 a_{20}+b_{11}=4 \lambda \mu$ and making the transformation $\xi=\lambda x, \eta=\lambda y+\frac{1}{4}\left(2 a_{20}-b_{11}\right) \lambda x^{2}$, we obtain the mentioned result.

From (2.2), system (2.1) becomes the following real autonomous planar system

$$
\begin{aligned}
& \frac{d x}{d t}=y+\mu x^{2}+\sum_{i+2 j=3}^{\infty} a_{i j} x^{i} y^{j}=X(x, y), \\
& \frac{d y}{d t}=-2 x^{3}+2 \mu x y+\sum_{i+2 j=4}^{\infty} b_{i j} x^{i} y^{j}=Y(x, y) .
\end{aligned}
$$

Write that

$$
X(x, y)=y+\sum_{k=2}^{\infty} X_{k}(x, y), Y(x, y)=\sum_{k=2}^{\infty} Y_{k}(x, y)
$$

where for $k=1,2, \cdots$,

$$
X_{k}(x, y)=\sum_{i+j=k} a_{i j} x^{i} y^{j}, Y_{k}(x, y)=\sum_{i+j=k} b_{i j} x^{i} y^{j} .
$$

By using the transformation of generalized polar coordinates

$$
x=r \cos \theta, \quad y=r^{2} \sin \theta,
$$

system (2.3) becomes

$$
\begin{aligned}
& \frac{d r}{d t}=\frac{\cos \theta\left[\sin \theta\left(1-2 \cos ^{2} \theta\right)+\mu\left(\cos ^{2} \theta+2 \sin ^{2} \theta\right)\right]}{1+\sin ^{2} \theta} r^{2}+o\left(r^{2}\right) \\
& \frac{d \theta}{d t}=\frac{-r}{2\left(1+\sin ^{2} \theta\right)\left(\cos ^{4} \theta+\sin ^{2} \theta\right)}+o(r)
\end{aligned}
$$

Thus, we have

$$
\frac{d r}{d \theta}=\frac{-\cos \theta\left[\sin \theta\left(1-2 \cos ^{2} \theta\right)+\mu\left(\cos ^{2} \theta+2 \sin ^{2} \theta\right)\right]}{2\left(\cos ^{4} \theta+\sin ^{2} \theta\right)} r+o(r)
$$

Let

$$
r=\tilde{r}(\theta, h)=\sum_{k=1}^{\infty} v_{k}(\theta) h^{k}
$$


be a solution of (2.5) satisfying the initial condition $\left.r\right|_{\theta=0}=h$, where $h$ is small and

$$
\begin{aligned}
v_{1}(\theta) & =\left(\cos ^{4} \theta+\sin ^{2} \theta\right)^{\frac{-1}{4}} \exp \left(\frac{-\mu}{2} \arctan \frac{\sin \theta}{\cos ^{2} \theta}\right), \\
v_{1}(k \pi) & =1, \quad k=0, \pm 1, \pm 2, \cdots .
\end{aligned}
$$

Because for all sufficiently small $r$, we have $d \theta / d t<0$. In a small neighborhood, we can define the successor function of system (2.3) as follows:

$$
\Delta(h)=\tilde{r}(-2 \pi, h)-h=\sum_{k=2}^{\infty} v_{k}(-2 \pi) h^{k} .
$$

We have the following result:

Lemma 2.3. For any positive integer $m, v_{2 m+1}(-2 \pi)$ has the form

$$
v_{2 m+1}(-2 \pi)=\sum_{k=1}^{m} \zeta_{k}^{(m)} v_{2 k}(-2 \pi)
$$

where $\zeta_{k}^{(m)}$ is a polynomial of $v_{j}(\pi), v_{j}(2 \pi), v_{j}(-2 \pi),(j=2,3, \cdots, 2 m)$ with rational coefficients.

It is differential from the center-focus problem for the elementary critical points, we know from Lemma 2.3 that when $k>1$ for the first non-zero $v_{k}(-2 \pi), k$ is an even integer.

\section{Definition 2.4.}

1. For any positive integer $m, v_{2 m}(-2 \pi)$ is called the $m$-th focal value of system (2.3) in the origin.

2. If $v_{2}(-2 \pi) \neq 0$, then, the origin of system (2.3) is called 1-order weakened focus. In addition, if there is an integer $m>1$, such that $v_{2}(-2 \pi)=v_{4}(-2 \pi)=\cdots=v_{2 m-2}(-2 \pi)=0$, but $v_{2} m(-2 \pi) \neq 0$, then the origin is called an m-order weakened focus of system (2.3).

3. If for all positive integers $m$, we have $v_{2 m}(-2 \pi)=0$, then the origin of system (2.3) is called a center.

We give the following key results, which define the quasi-Lyapunov constants and provide a way of computing them.

Theorem 2.5. For system (2.3), one can construct successively a formal series

$$
M(x, y)=y^{2}+\sum_{k+j=3}^{\infty} c_{k j} x^{k} y^{j}
$$

such that

$$
\frac{\partial}{\partial x}\left(\frac{X}{M^{s+1}}\right)+\frac{\partial}{\partial y}\left(\frac{Y}{M^{s+1}}\right)=\frac{1}{M^{s+2}} \sum_{m=1}^{\infty}(2 m-4 s-1) \lambda_{m} x^{2 m+4},
$$

i.e.,

$$
\left(\frac{\partial X}{\partial x}+\frac{\partial Y}{\partial y}\right) M-(s+1)\left(\frac{\partial M}{\partial x} X+\frac{\partial M}{\partial y} Y\right)=\sum_{m=1}^{\infty} \lambda_{m}(2 m-4 s-1) x^{2 m+4},
$$

where $\mathrm{s}$ is a given positive integer,

$$
c_{30}=0, \quad c_{40}=1
$$

and

$$
\left\{v_{2 m}(-2 \pi)\right\} \sim\left\{\sigma_{m} \lambda_{m}\right\}
$$

with

$$
\sigma_{\mathrm{m}}=\frac{1}{2} \int_{0}^{2 \pi} \frac{\left(1+\sin ^{2} \theta\right) \cos ^{2 m+4} \theta}{\left(\cos ^{4} \theta+\sin ^{2} \theta\right)^{2}} v_{1}^{2 m-1}(\theta) \mathrm{d} \theta>0
$$


We see from (2.6) and (2.8) that when (2.4) holds, $M=y^{2}+x^{4}+o\left(r^{4}\right)$.

Definition 2.6. For system (2.3), $\lambda_{m}$ is called the $m$-th quasi-Lyapunov constant of the origin.

Theorem 2.7. For any positive integer s and a given number sequence

$$
\left\{c_{0 \beta}\right\}, \beta \geqslant 3,
$$

one can construct successively the terms with the coefficients $c_{\alpha \beta}$ satisfying $\alpha \neq 0$ of the formal series

$$
M(x, y)=y^{2}+\sum_{\alpha+\beta=3}^{\infty} c_{\alpha \beta} x^{\alpha} y^{\beta}=\sum_{k=2}^{\infty} M_{k}(x, y),
$$

such that

$$
\frac{\partial}{\partial x}\left(\frac{X}{M^{s+1}}\right)+\frac{\partial}{\partial y}\left(\frac{Y}{M^{s+1}}\right)=\frac{1}{M^{s+2}} \sum_{m=5}^{\infty} \omega_{m}(s, \mu) x^{m},
$$

where for all $k, M_{k}(x, y)$ is a $k$-homogeneous polynomial of $x, y$ and $s \mu=0$.

Now, (2.10) can be written by

$$
\left(\frac{\partial X}{\partial x}+\frac{\partial Y}{\partial y}\right) M-(s+1)\left(\frac{\partial M}{\partial x} X+\frac{\partial M}{\partial y} Y\right)=\sum_{m=3}^{\infty} \omega_{m}(s, \mu) x^{m} .
$$

It is easy to see that (2.11) is linear with respect to the function $M$, so that we can easily find the following recursive formulae for the calculation of $c_{\alpha \beta}$ and $\omega_{m}(s, \mu)$.

Theorem 2.8. For $\alpha \geqslant 1, \alpha+\beta \geqslant 3$ in (2.9) and (2.10), $c_{\alpha \beta}$ can be uniquely determined by the recursive formula

$$
c_{\alpha \beta}=\frac{1}{(s+1) \alpha}\left(A_{\alpha-1, \beta+1}+B_{\alpha-1, \beta+1}\right) \text {. }
$$

For $m \geqslant 1, \omega_{m}(s, \mu)$ can be uniquely determined by the recursive formula

$$
\omega_{m}(s, \mu)=A_{m, 0}+B_{m, 0},
$$

where

$$
\begin{aligned}
& A_{\alpha \beta}=\sum_{k+j=2}^{\alpha+\beta-1}[k-(s+1)(\alpha-k+1)] a_{k j} c_{\alpha-k+1, \beta-j}, \\
& B_{\alpha \beta}=\sum_{k+j=2}^{\alpha+\beta-1}[j-(s+1)(\beta-j+1)] b_{k j} c_{\alpha-k, \beta-j+1} .
\end{aligned}
$$

Notice that in (2.12), we set

$$
\begin{aligned}
c_{00} & =c_{10}=c_{01}=0, \\
c_{20} & =c_{11}=0, \quad c_{02}=1, \\
c_{\alpha \beta} & =0, \text { if } \alpha<0 \text { or } \beta<0 .
\end{aligned}
$$

We see from Theorem 2.8 that, by choosing $\left\{\mathrm{c}_{\alpha \beta}\right\}$, such that

$$
\omega_{2 k+1}(s, \mu)=0, \quad k=1,2, \cdots,
$$

we can obtain a solution group of $\left\{c_{\alpha \beta}\right\}$ of (2.13), thus, we have

$$
\lambda_{\mathrm{m}}=\frac{\omega_{2 \mathrm{~m}+4}(\mathrm{~s}, \mu)}{2 \mathrm{~m}-4 \mathrm{~s}-1} .
$$

Clearly, the recursive formulae presented by Theorem 2.8 is linear with respect to all $c_{\alpha \beta}$. Accordingly, it is convenient to realize the computations of quasi-Lyapunov constants by using computer algebraic system like Mathematica. 
Theorem 2.9. If $\mu \neq 0$, the origin of system (2.3) is not an analytic center.

Theorem 2.10. The origin of system (2.3) is an analytic center if and only if there exists an analytic first integral $\mathrm{F}(\mathrm{x}, \mathrm{y})$ in the neighborhood of the origin, which is the power series

$$
F(x, y)=x^{4}+y^{2}+\sum_{k+2 j=5}^{\infty} C_{k j} x^{k} y^{j} .
$$

Theorem 2.11. If the origin of system (2.3) is a nilpotent center, and system (2.3) is symmetric with respect to the origin, namely,

$$
\begin{aligned}
& X(-x,-y)=-X(x, y), \\
& Y(-x,-y)=-Y(x, y),
\end{aligned}
$$

then the origin of system (2.3) is an analytic center.

Theorem 2.12. If system (2.3) is symmetric with respect to the the x-axis, then the origin of system (2.3) is an analytic center.

Consider the system

$$
\begin{aligned}
& \frac{d x}{d t}=\delta x+y+\sum_{k+j=2}^{\infty} a_{k j}(\gamma) x^{k} y^{j}, \\
& \frac{d y}{d t}=2 \delta y+\sum_{k+j=2}^{\infty} b_{k j}(\gamma) x^{k} y^{j},
\end{aligned}
$$

where $\gamma=\left\{\gamma_{1}, \gamma_{2}, \cdots, \gamma_{m-1}\right\}$ is $(m-1)$-dimensional parameter vector. Let $\gamma_{0}=\left\{\gamma_{1}^{(0)}, \gamma_{2}^{(0)}, \ldots, \gamma_{m-1}^{(0)}\right\}$ be a point at the parameter space. Suppose that for $\left\|\gamma-\gamma_{0}\right\| \ll 1$, the functions of the right hand of system (2.14) are power series of $x, y$ with a non-zero convergence radius and have continuous partial derivatives with respect to $\gamma$. In addition,

$$
a_{20}(\gamma) \equiv \mu, \quad b_{20}(\gamma) \equiv 0, \quad b_{11}(\gamma) \equiv 2 \mu, \quad b_{30}(\gamma) \equiv-2 .
$$

For an integer $k$, let $v_{2 k}(-2 \pi, \gamma)$ be the k-order focal value of the origin of system $(2.14)_{\delta=0}$.

Theorem 2.13. If for $\gamma=\gamma_{0}$, the origin of system $(2.14)_{\delta=0}$ is an m-order weak focus, and the Jacobian

$$
\left.\frac{\partial\left(v_{2}, v_{4}, \cdots, v_{2 m-2}\right)}{\partial\left(\gamma_{1}, \gamma_{2}, \cdots, \gamma_{m-1}\right)}\right|_{\gamma=\gamma_{0}} \neq 0
$$

then there exist two positive number $\delta^{*}$ and $\gamma^{*}$, such that for $0<|\delta|<\delta^{*}, 0<\left\|\gamma-\gamma_{0}\right\|<\gamma^{*}$, in a neighborhood of the origin, system (2.14) has at most $m$ limit cycles which enclose the origin (an elementary node) $\mathrm{O}(0,0)$. In addition, under the above conditions, there exist $\tilde{\gamma}, \tilde{\delta}$, such that when $\gamma=\tilde{\gamma}, \delta=\tilde{\delta}$, there exist exactly m limit cycles of (2.14) in a small neighborhood of the origin.

\section{Quasi-Lyapunov constants and analytic center conditions}

In this section we will derive the analytic center conditions for the origin of system (1.1).

Performing the transformation

$$
x_{1}=x, \quad y_{1}=\frac{1}{\sqrt{n}} y^{n}, \quad d t_{1}=\sqrt{n} y^{n-1} d t
$$

and still using $(x, y, t)$ to represent $\left(x_{1}, y_{1}, t_{1}\right)$, system (1.1) becomes

$$
\begin{aligned}
& \frac{d x}{d t}=y+a_{41} x^{4} y+\sqrt{n} a_{32} x^{3} y^{2}+n a_{23} x^{2} y^{3}+n^{\frac{3}{2}} a_{14} x y^{4}+n^{2} a_{05} y^{5}, \\
& \frac{d y}{d t}=-2 x^{3}+b_{50} x^{5}+\sqrt{n} b_{41} x^{4} y+n b_{32} x^{3} y^{2}+n^{\frac{3}{2}} b_{23} x^{2} y^{3}+n^{2} b_{14} x y^{4}+n^{\frac{5}{2}} b_{05} y^{5} .
\end{aligned}
$$

Now we start the preparation of computing quasi-Lyapunov constants at the origin of system (3.1). 
Lemma 3.1. Assume that $\mathrm{s}$ is a natural number. We can derive a power series (2.6) for system (3.1) under which (2.7) is satisfied, where

$$
\mathrm{c}_{0,0}=0, \quad \mathrm{c}_{1,0}=0, \quad \mathrm{c}_{0,1}=0, \quad \mathrm{c}_{2,0}=0, \quad \mathrm{c}_{1,1}=0, \quad \mathrm{c}_{0,2}=1,
$$

in addition, for any natural numbers $\alpha, \beta, \mathrm{c}_{\alpha, \beta}$ is given by the following recursive formula

$$
\begin{aligned}
c_{\alpha, \beta}= & \left(-b_{50}(1+s)(2+\beta) c_{-6+\alpha, 2+\beta}+b_{41} \sqrt{n}(1-(1+s)(1+\beta)) c_{-5+\alpha, 1+\beta}\right. \\
& +a_{41}(4-(1+s)(-4+\alpha)) c_{-4+\alpha, \beta}+b_{32} n(2-(1+s) \beta) c_{-4+\alpha, \beta} \\
& +2(1+s)(2+\beta) c_{-4+\alpha, 2+\beta}+a_{32} \sqrt{n}(3-(1+s)(-3+\alpha)) c_{-3+\alpha,-1+\beta} \\
& +b_{23} n^{\frac{3}{2}}(3-(1+s)(-1+\beta)) c_{-3+\alpha,-1+\beta}+a_{23} n(2-(1+s)(-2+\alpha)) c_{-2+\alpha,-2+\beta} \\
& +b_{14} n^{2}(4-(1+s)(-2+\beta)) c_{-2+\alpha,-2+\beta}+a_{14} \mathrm{n}^{\frac{3}{2}}(1-(1+s)(-1+\alpha)) c_{-1+\alpha,-3+\beta} \\
& \left.+b_{05} n^{\frac{5}{2}}(5-(1+s)(-3+\beta)) c_{-1+\alpha,-3+\beta}-a_{05} n^{2}(1+s) \alpha c_{\alpha,-4+\beta}\right) /(s+1) / \alpha,
\end{aligned}
$$

and, for any natural number $\mathrm{m}, \omega_{\mathrm{m}}$ is given by the following recursive formula

$$
\begin{aligned}
w_{m}= & -b_{50}(1+s) c_{-5+m, 1}+b_{41} \sqrt{n} c_{-4+m, 0}+b_{32} n(3+s) c_{-3+m,-1} \\
& +a_{41}(4-(-3+m)(1+s)) c_{-3+m,-1}+2(1+s) c_{-3+m, 1} \\
& +b_{23} n^{\frac{3}{2}}(3+2(1+s)) c_{-2+m,-2}+a_{32} \sqrt{n}(3-(-2+m)(1+s)) c_{-2+m,-2} \\
& +b_{14} n^{2}(4+3(1+s)) c_{-1+m,-3}+a_{23} n(2-(-1+m)(1+s)) c_{-1+m,-3} \\
& +b_{05} n^{\frac{5}{2}}(5+4(1+s)) c_{m,-4}+a_{14} n^{\frac{3}{2}}(1-m(1+s)) c_{m,-4}-a_{05}(1+m) n^{2}(1+s) c_{1+m,-5} .
\end{aligned}
$$

Applying Lemma 3.1 and computing with Mathematica, we have:

Theorem 3.2. The first seven quasi-Lyapunov constants at the origin of system (3.1) are as follows:

$$
\begin{aligned}
& \lambda_{1}=0, \\
& \lambda_{2} \sim \frac{1}{5} \sqrt{n} b_{41}, \\
& \lambda_{3} \sim \frac{2}{7} \sqrt{n}\left(a_{32}+n b_{23}\right), \\
& \lambda_{4} \sim \frac{4}{15} n^{\frac{3}{2}}\left(a_{14}+5 n b_{05}\right), \\
& \lambda_{5} \sim \frac{4}{77} n^{\frac{3}{2}} b_{23}\left(2 a_{41}+n b_{32}\right),
\end{aligned}
$$

and, either

$$
\begin{aligned}
& \lambda_{6} \sim \frac{8}{39} n^{\frac{5}{2}} b_{05}\left(2 a_{41}+n b_{32}\right), \\
& \lambda_{7} \sim \frac{16}{33} n^{\frac{7}{2}} b_{05}\left(a_{23}+2 n b_{14}\right),
\end{aligned}
$$

for $\mathrm{b}_{23}=0$, or

$$
\begin{aligned}
& \lambda_{6} \sim \frac{8}{117} \mathrm{n}^{\frac{5}{2}} \mathrm{~b}_{23}\left(\mathrm{a}_{23}+2 \mathrm{nb_{14 }}\right), \\
& \lambda_{7} \sim 0,
\end{aligned}
$$

for $2 a_{41}+n b_{32}=0, b_{23} \neq 0$. In the above expression of $\lambda_{k}$, it is assumed that $\lambda_{1}=\lambda_{2}=\cdots=\lambda_{k-1}=0, k=$ $2,3,4,5,6,7$.

An immediate consequence of Theorem 3.2 is as follows.

Theorem 3.3. The first seven quasi-Lypaunov constants at the origin of system (3.1) vanish if and only if the coefficients satisfy one of the following two sets of conditions:

$$
\begin{gathered}
b_{41}=a_{32}=a_{14}=b_{23}=b_{05}=0, \\
b_{41}=a_{32}+n b_{23}=a_{14}+5 n b_{05}=2 a_{41}+n b_{32}=a_{23}+2 n b_{14}=0 .
\end{gathered}
$$


Proof. Notice expressions (3.2) and (3.3), from the vanishing of the first seven quasi-Lyapunov constants, it follows one of the following conditions:

$$
\begin{gathered}
b_{41}=a_{32}=a_{14}=b_{23}=b_{05}=0, \\
b_{41}=a_{32}=b_{23}=a_{14}+5 n b_{05}=2 a_{41}+n b_{32}=a_{23}+2 n b_{14}=0
\end{gathered}
$$

Analogously, notice expressions (3.2) and (3.4), from the vanishing of the first seven quasi-Lyapunov constants, it follows one of the following conditions:

$$
b_{41}=a_{32}+n b_{23}=a_{14}+5 n b_{05}=2 a_{41}+n b_{32}=a_{23}+2 n b_{14}=0, \quad b_{23} \neq 0 .
$$

Conditions (3.7) and (3.8) can be explicitly united as condition (3.6). Therefore, the claim is proved.

The next result assures the analytic center conditions.

Theorem 3.4. System (3.1) has an analytic center at the origin if and only if condition (3.5) or (3.6) is satisfied.

Proof. When condition (3.5) holds, system (3.1) is transformed into

$$
\begin{aligned}
& \frac{d x}{d t}=y\left(1+a_{41} x^{4}+n a_{23} x^{2} y^{2}+n^{2} a_{05} y^{4}\right), \\
& \frac{d y}{d t}=x\left(-2 x^{2}+b_{50} x^{4}+n b_{32} x^{2} y^{2}+n^{2} b_{14} y^{4}\right),
\end{aligned}
$$

whose vector field is symmetric with respect to the origin.

When condition (3.6) is satisfied, system (3.1) is transformed into

$$
\begin{aligned}
& \frac{d x}{d t}=\frac{1}{2} y\left(2-n b_{32} x^{4}-2 n^{\frac{3}{2}} b_{23} x^{3} y-4 n^{2} b_{14} x^{2} y^{2}-10 n^{\frac{5}{2}} b_{05} x y^{3}+2 n^{2} a_{05} y^{4}\right), \\
& \frac{d y}{d t}=-2 x^{3}+b_{50} x^{5}+n b_{32} x^{3} y^{2}+n^{\frac{3}{2}} b_{23} x^{2} y^{3}+n^{2} b_{14} x y^{4}+n^{\frac{5}{2}} b_{05} y^{5},
\end{aligned}
$$

which is Hamiltonian and has an analytic first integral defined in the neighborhood of the nilpotent center

$$
F_{1}(x, y)=x^{4}+y^{2}-\frac{1}{3} b_{50} x^{6}-\frac{1}{2} n b_{32} x^{4} y^{2}-\frac{2}{3} n^{\frac{3}{2}} b_{23} x^{3} y^{3}-n^{2} b_{14} x^{2} y^{4}-2 n^{\frac{5}{2}} b_{05} x y^{5}+\frac{1}{3} n^{2} a_{05} y^{6} .
$$

Therefore, the theorem follows.

As a matter of fact, when condition (3.5) holds, system (1.1) goes over to

$$
\begin{aligned}
& \frac{d x}{d t}=y^{2 n-1}\left(1+a_{41} x^{4}+a_{23} x^{2} y^{2 n}+a_{05} y^{4 n}\right), \\
& \frac{d y}{d t}=x\left(-2 x^{2}+b_{50} x^{4}+b_{32} x^{2} y^{2 n}+b_{14} y^{4 n}\right),
\end{aligned}
$$

whose vector field is symmetric with respect to the origin.

When condition (3.6) holds, system (1.1) goes over to

$$
\begin{aligned}
& \frac{d x}{d t}=\frac{1}{2} y^{2 n-1}\left(2-n b_{32} x^{4}-2 n b_{23} x^{3} y^{n}-4 n b_{14} x^{2} y^{2 n}-10 n b_{05} x y^{3 n}+2 a_{05} y^{4 n}\right), \\
& \frac{d y}{d t}=-2 x^{3}+b_{50} x^{5}+b_{32} x^{3} y^{2 n}+b_{23} x^{2} y^{3 n}+b_{14} x y^{4 n}+b_{05} y^{5 n},
\end{aligned}
$$

which is Hamiltonian. Going back through the change of variables we find that, in a neighborhood of the origin, the analytic function

$$
F_{2}(x, y)=x^{4}+\frac{1}{n} y^{2 n}-\frac{1}{3} b_{50} x^{6}-\frac{1}{2} b_{32} x^{4} y^{2 n}-\frac{2}{3} b_{23} x^{3} y^{3 n}-b_{14} x^{2} y^{4 n}-2 b_{05} x y^{5 n}+\frac{1}{3 n} a_{05} y^{6 n}
$$

is a first integral of system (3.9).

Thus, we obtain the following:

Corollary 3.5. System (1.1) has an analytic center at the origin if and only if condition (3.5) or (3.6) is satisfied. 


\section{Limit cycle bifurcation}

In this section, we shall establish the condition under which $\mathrm{O}(0,0)$ is at most a seven-order weak focus. Additionally, we will prove the perturbed system of (3.1) can produce five limit cycles enclosing an elementary node at the origin.

It follows from $\lambda_{1}=\lambda_{2}=\lambda_{3}=\lambda_{4}=\lambda_{5}=\lambda_{6}=0, \lambda_{7} \neq 0$ that

Theorem 4.1. The origin of system (3.1) is a seven-order weak focus if and only if

$$
b_{41}=a_{32}=b_{23}=0, \quad a_{14}=-5 n b_{05}, \quad a_{41}=-\frac{n}{2} b_{32}, \quad b_{05}\left(a_{23}+2 n b_{14}\right) \neq 0 .
$$

Consider the perturbed system of (3.1)

$$
\begin{aligned}
& \frac{d x}{d t}=\delta(\varepsilon) x+y+a_{41}(\varepsilon) x^{4} y+\sqrt{n} a_{32}(\varepsilon) x^{3} y^{2}+n a_{23} x^{2} y^{3}+n^{\frac{3}{2}} a_{14}(\varepsilon) x y^{4}+n^{2} a_{05} y^{5}, \\
& \frac{d y}{d t}=2 \delta(\varepsilon) y-2 x^{3}+b_{50} x^{5}+\sqrt{n} b_{41}(\varepsilon) x^{4} y+n b_{32} x^{3} y^{2}+n^{\frac{3}{2}} b_{23} x^{2} y^{3}+n^{2} b_{14} x y^{4}+n^{\frac{5}{2}} b_{05} y^{5} .
\end{aligned}
$$

In order to get five limit cycles, we only need to show that, when condition (4.1) holds, the Jacobian of $\left(\lambda_{2}, \lambda_{3}, \lambda_{4}, \lambda_{6}\right)$ with respect to $\left(b_{41}, a_{32}, a_{14}, a_{41}\right)$ does not vanish. An easy computation shows that

$$
\left.\frac{\partial\left(\lambda_{2}, \lambda_{3}, \lambda_{4}, \lambda_{6}\right)}{\partial\left(b_{41}, a_{32}, a_{14}, a_{41}\right)}\right|_{(4.1)}=\frac{128}{20475} n^{5} b_{05} \neq 0
$$

The above discussions indicate the following.

Theorem 4.2. If the origin of system (3.1), (1.1) is a weak focus of order seven, for $0<\delta \ll 1$, making a small perturbation to the coefficient group $\left(\mathrm{b}_{41}, \mathrm{a}_{32}, \mathrm{a}_{14}, \mathrm{a}_{41}\right)$, then for system (4.2), in a small neighborhood of the origin, there exist exactly five small amplitude limit cycles enclosing the origin $\mathrm{O}(0,0)$, which is an elementary node.

Example 4.3. Take

$$
\begin{aligned}
& \delta(\varepsilon)=-\varepsilon^{56}, \quad b_{41}(\varepsilon)=\varepsilon^{30}, \quad a_{32}(\varepsilon)=-\varepsilon^{20}, \\
& a_{14}(\varepsilon)=-5 n c_{05} \operatorname{sign}\left(c_{05}\right)+\epsilon^{12}, \quad a_{41}(\varepsilon)=-\frac{1}{2} n c_{32} \operatorname{sign}\left(c_{32}\right)-\varepsilon^{2}, \\
& b_{05}=c_{05} \operatorname{sign}\left(c_{05}\right), \quad b_{32}=c_{32} \operatorname{sign}\left(c_{32}\right), \quad a_{23}=c_{23} \operatorname{sign}\left(c_{23}\right), \quad b_{14}=c_{14} \operatorname{sign}\left(c_{14}\right), \quad b_{23}=0,
\end{aligned}
$$

where $c_{05}, c_{32}, c_{23}, c_{14}$ are arbitrary nonzero real constants.

Taking into account expressions (3.2) and (3.3), straightforward computations give the first seven quasi-Lyapunov constants at the origin of system (4.2):

$$
\begin{aligned}
& \lambda_{1}=\mathrm{o}\left(\varepsilon^{42}\right), \\
& \lambda_{2} \sim \frac{1}{5} \sqrt{n} \varepsilon^{30}+o\left(\varepsilon^{30}\right), \\
& \lambda_{3} \sim-\frac{2}{7} \sqrt{n} \varepsilon^{20}+o\left(\varepsilon^{20}\right) \text {, } \\
& \lambda_{4} \sim \frac{4}{15} \mathrm{n}^{\frac{3}{2}} \varepsilon^{12}+\mathrm{o}\left(\varepsilon^{12}\right), \\
& \lambda_{5} \sim \mathrm{o}\left(\varepsilon^{6}\right) \text {, } \\
& \lambda_{6} \sim-\frac{16}{39} n^{\frac{5}{2}} c_{05} \operatorname{sign}\left(\mathrm{c}_{05}\right) \varepsilon^{2}+o\left(\varepsilon^{2}\right), \\
& \lambda_{7} \sim \frac{16}{33} \mathrm{n}^{\frac{7}{2}} \mathrm{c}_{05} \operatorname{sign}\left(\mathrm{c}_{05}\right)\left(\mathrm{c}_{23} \operatorname{sign}\left(\mathrm{c}_{23}\right)+2 \mathrm{nc}_{14} \operatorname{sign}\left(\mathrm{c}_{14}\right)\right)+\mathrm{o}(1) .
\end{aligned}
$$

Then, for $0<\varepsilon \ll 1$, system (4.2) has five limit cycles $\Gamma_{k}: r=\tilde{r}\left(\theta, h_{k}(\varepsilon)\right)$ in a small neighborhood of the origin, where $h_{k}(\varepsilon)=O\left(\varepsilon^{k}\right), k=2,3,4,6,7$.

\section{Acknowledgment}

This work is supported in part by the National Nature Science Foundation of China (No. 11101126), Scientific Research Foundation for Doctoral Scholars of HAUST (No. 13480021) and the Foundation for Youth Leaders of Disciplines in Science of HAUST (No. 13490003). 


\section{References}

[1] V. V. Amelkin, N. A. Lukashevich, A. P. Sadovskiü, Nelineı̆nye kolebaniya v sistemakh vtorogo poryadka, (Russian) [[Nonlinear oscillations in second-order systems]] Beloruss. Gos. Univ., Minsk, (1982), 208 pages. 2, 2

[2] A. F. Andreev, Investigation of the behaviour of the integral curves of a system of two differential equations in the neighbourhood of a singular point, Amer. Math. Soc. Transl., 8 (1958), 183-207. 1

[3] M. Berthier, R. Moussu, Réversibilité et classification des centres nilpotents, (French) [[Reversibility and classification of nilpotent centers]] Ann. Inst. Fourier (Grenoble), 44 (1994), 465-494. 1

[4] J. Écalle, Introduction aux fonctions analysables et preuve constructive de la conjecture de Dulac, (French) [[Introduction to analyzable functions and constructive proof of the Dulac conjecture]] Actualités Mathématiques, [Current Mathematical Topics] Hermann, Paris, (1992). 1

[5] I. A. García, J. Giné, Analytic nilpotent centers with analytic first integral, Nonlinear Anal., 72 (2010), 3732-3738. 1

[6] H. Giacomini, J. Giné, J. Llibre, The problem of distinguishing between a center and a focus for nilpotent and degenerate analytic systems, J. Differential Equations, 227 (2006), 406-426. 1

[7] H. Giacomini, J. Giné, J. Llibre, Corrigendum to: "The problem of distinguishing between a center and a focus for nilpotent and degenerate analytic systems", J. Differential Equations, 232 (2007), 702. 1

[8] J. Giné, Analytic integrability and characterization of centers for generalized nilpotent singular points, Appl. Math. Comput., 148 (2004), 849-868. 1

[9] M.-A. Han, V. G. Romanovski, Limit cycle bifurcations from a nilpotent focus or center of planar systems, Abstr. Appl. Anal., 2012 (2012), 28 pages. 1

[10] F. Li, Y.-Y. Liu, Limit cycles in a class of switching system with a degenerate singular point, Chaos Solitons Fractals, 92 (2016), 86-90. 1

[11] F. Li, P. Yu, Y.-R. Liu, Analytic integrability of two lopsided systems, Internat. J. Bifur. Chaos Appl. Sci. Engrg., 26 (2016), 14 pages. 1

[12] F. Li, P. Yu, Y. Tian, Y.-R. Liu, Center and isochronous center conditions for switching systems associated with elementary singular points, Commun. Nonlinear Sci. Numer. Simul., 28 (2015), 81-97. 1

[13] Y.-R. Liu, J.-B. Li, On third-order nilpotent critical points: integral factor method, Internat. J. Bifur. Chaos Appl. Sci. Engrg., 21 (2011), 1293-1309. 1, 2

[14] T. Liu, L.-G. Wu, F. Li, Analytic center of nilpotent critical points, Internat. J. Bifur. Chaos Appl. Sci. Engrg., 22 (2012), 8 pages. 1,2

[15] R. Moussu, Symétrie et forme normale des centres et foyers dégénérés, (French) [[Symmetry and normal form in degenerate centers and foci]] Ergodic Theory Dynamical Systems, 2 (1982), 241-251. 1

[16] A.P. Sadovskii, Problem of distinguishing a center and a focus for a system with a nonvanishing linear part, translated from Differ. Uravn., 12 (1976), 1238-1246. 1

[17] F. Takens, Singularities of vector fields, Inst. Hautes Études Sci. Publ. Math., 43 (1974), 47-100. 1

[18] M. A. Teixeira, J.-Z. Yang, The center-focus problem and reversibility, J. Differential Equations, 174 (2001), 237-251. 1 